\title{
Design and Motion Analysis of Dynamic Wire-Controlled Hanging Plate Balancing Mechanism in Well
}

\author{
Yuhang $\mathrm{He}^{1,2}$, Xiaoshuang $\mathrm{Liu}^{1,2}$ and DW Tang ${ }^{1,2,{ }^{*}}$ \\ ${ }^{1}$ School of mechanical engineering, The University of South China, Hunan, China \\ ${ }^{2}$ Hunan nuclear fuel cycle technology and equipment collaborative innovation center, Hunan, China \\ ${ }^{*}$ Corresponding author
}

Keywords: Hanging plate balancing mechanism, Force feedback balance, Inverse Kinematics, Cable module, Attitude balance, Tension equilibrium.

\begin{abstract}
Aiming at the problems of sway and collision caused by tilt, swing and uneven distribution of wire rope tension in the hoisting process of hoisting in the well, this paper designs a control system of wire rope tension balance and attitude adjustment of the hoisting disk based on the inverse kinematics model and the force feedback balance model. Through the position information of the hanging plate, the wire rope displacement information and the wire rope force feedback information to adjust the length and tension of the wire rope, and the finite element software Adams is used to establish the lifting plate balance mechanism pulley sling model, which simulates the lifting plate during the lifting movement. The attitude leveling and tension balancing process. Simulation results show that: in the process of lifting the sling, the wire control sling balancing mechanism can quickly level the sling and effectively reduce the swing of the sling.
\end{abstract}

\section{Introduction}

At present, lifting equipment is mainly divided into four types: light and small lifting equipment, bridge type, boom type and cable type according to the structure.Flexible steel wire rope not only has high tensile strength, but also has good receiving capacity, which is convenient for storage and transportation. However, in the operation platform of multi-rope synchronous hoisting, the swing of flexible steel wire rope and uneven force often lead to the tilt of the operation platform, which may easily lead to the safety accident of the collision between the hoisting platform and the shaft wall. In recent years, a lot of research work has been done on the leveling and anti-sway lifting of vertical lifting platforms in China, and some research results have been obtained.

On the leveling problem of the platform, Zhu Bao [1] used the spatial geometric decomposition method to establish a spatial model and derived a horizontal decomposition algorithm, which would automatically level the rotary platform without re-adjusting the sensitive orientation of the horizontal sensor. Zhai yujian[2] and zhang fang[3] both adopted multi-point support platform for attitude leveling, and established PLC control system for leveling by controlling Angle error and position error analysis. Yi jianqiang [4] designed a fuzzy controller by using the four-rope suspension mechanism, which can adjust and even the tension of the suspension platform. Shao Xingguo [5] designed the attitude leveling and wire rope tension equalization controller based on the inverse kinematics model, and realized the attitude leveling and tension balance of the hanging plate by adjusting the running speed of the four stable motor. Zhang Fuming [6] uses the PLC control system to synchronously lift and suspend the five lifting points on the disc. In the anti-swing aspect of the platform, Du Wenzheng [7], Wang Xiaojun [8] designed a method for crane positioning and anti-sway based on fuzzy control. Wang Keqi [9] regards the crane as a non-linear coupling system with insufficient excitation, decoupling and linearizing its working position and rope length, and designing a full-state feedback controller for anti-swing. Although the research work of the predecessors improved the swinging and tilting problems of the vertical working platform, it took a long time to adjust the response of the platform. In the narrow working environment of the well, it was unable to respond to the tilting and swinging of the platform in time. Aiming at this problem, based on the inverse kinematics model and force feedback balance model 
of the hanging plate balance mechanism, the dynamic wire-controlled hanging plate balance mechanism in the well is designed to improve the response time of the hanging plate leveling.

\section{Design and Analysis of Crane Structure}

The principle prototype of the dynamic wire-controlled feedback balance hoisting mechanism developed for the narrow well in the radiation environment is shown in Fig.1, which is mainly composed of five parts: mobile mechanism, lifting mechanism, wire-controlled mechanism, lifting tray and control system. The wire control mechanism is mounted on the moving mechanism of the bridge, and can perform linear motion on the moving track of the bridge. One end of the wire rope is connected with the wire control mechanism, and the other end is connected with the circular hanging plate.

The geometrical parameters of the working area of the mechanism are as follows: the wellhead diameter of the storage weight is $650 \mathrm{~mm}$, its maximum depth is $14 \mathrm{~m}$, the diameter of the weight barrel is $600 \mathrm{~mm}$, the distance between the bucket wall and the well wall is small, and the lifting is carried out during the lifting process. The vibration performance requirements of the equipment are large, and the ability to prevent shaking during the lifting process of the heavy objects is required. Therefore, this paper adopts a rope lifting four-wire remote control lifting method. By adjusting the length of the wire control rope before lifting, the attitude of the hanging plate is leveled. After lifting, the tensioning force of the wire-controlled rope is changed to hinder the swing movement of the heavy bucket, so as to realize the stable lifting of the heavy bucket.

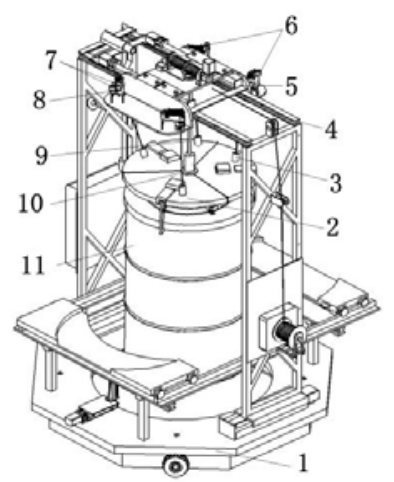

1-Walking mechanism; 2-The stage; 3-Tension sensor; 4-The frame; 5-Mobile mechanism; 6,7-Wire control agencies; 8-Pull rope displacement sensor; 9-The main bearing rope; 10-Wire rope; 11-Heavy barrels

Figure 1. The Line control and balance lifting mechanism diagram

\section{Anti-sway Motion Analysis}

\section{Wire Rope Length Description}

The sling is closely connected with the lifting rope. The middle sling is the main load bearing rope, and the four hanging ropes at the edge $a_{i} b_{i}(i=1,2,3,4)$ are line-controlled lifting ropes. The four moving points of $\mathrm{a}_{1}, \mathrm{a}_{2}, \mathrm{a}_{3}$ and $\mathrm{a}_{4}$ are distributed on the sling, and the coordinates are $\mathrm{a}_{1}\left(\mathrm{x}_{1}, \mathrm{y}_{1}, \mathrm{Z}_{1}\right)$, $\mathrm{a}_{2}\left(\mathrm{x}_{2}, \mathrm{y}_{2}, \mathrm{z}_{2}\right), \mathrm{a}_{3}\left(\mathrm{x}_{3}, \mathrm{y}_{3}, \mathrm{z}_{3}\right)$ and $\mathrm{a}_{4}\left(\mathrm{x}_{4}, \mathrm{y}_{4}, \mathrm{z}_{4}\right)$. The four points of $\mathrm{b}_{1}, \mathrm{~b}_{2}, \mathrm{~b}_{3}$ and $\mathrm{b}_{4}$ are fixed points distributed on the bridge-wire control mechanism. Their coordinates are known and are expressed as $\mathrm{b}_{1}\left(\mathrm{x}_{5}, \mathrm{y}_{5}, \mathrm{z}_{5}\right), \mathrm{b}_{2}\left(\mathrm{x}_{6}, \mathrm{y}_{6}, \mathrm{z}_{6}\right), \mathrm{b}_{3}\left(\mathrm{x}_{7}, \mathrm{y}_{7}, \mathrm{z}_{7}\right)$ and $\mathrm{b}_{4}\left(\mathrm{x}_{8}, \mathrm{y}_{8}, \mathrm{z}_{8}\right)$. The coordinates of the $\mathrm{a}_{\mathrm{i}}$ point can be represented by the spatial position $\mathrm{O}(\mathrm{x}, \mathrm{y}, \mathrm{z})$ of the center point of the hanging plate and the attitude transformation matrix $\mathrm{T}$ of the hanging plate, which is described in detail in the fourth part of the text.The length of the four ropes can be calculated from the coordinates of $\mathrm{a}_{1} \sim \mathrm{a} 4$ and the coordinates of $b_{1} \sim b_{4}$.

The initial length of the four wire ropes can be expressed as:

$$
l_{a_{i} b_{i}}=\sqrt{\left(x_{i}-x_{i+4}\right)^{2}+\left(y_{i}-y_{i+4}\right)^{2}+\left(z_{i}-z_{i+4}\right)^{2}}(i=1,2 、 3 、 4)
$$


The actual length of the wire rope $l_{i}(\mathrm{i}=1,2,3,4)$ can be expressed as:

$$
l_{\mathrm{i}}=l_{\mathrm{ab}}-\frac{R t \omega \pi}{180^{\circ}}
$$

Where lab represents the initial length of the wire control rope, $l_{i}$ represents the actual length of the wire control rope, $\omega$ represents the motor speed, $t$ represents time, $\mathrm{R}$ represents the motor radius of gyration.

\section{Wire Control Rope Force Analysis}

The mechanical force is decomposed on the pulling force $f_{l}$ of the wire control rope $l_{a b}$. As shown in Fig. 2, the length of the wire control rope is $l$, the distance between the moving point of the wire control rope and the fixed point of the wire control rope on the straight line is $s$, and the depth of the well is $h$.

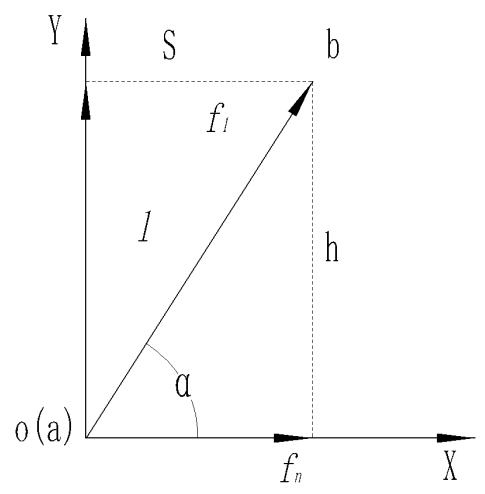

Figure 2. Force analysis of line control rope

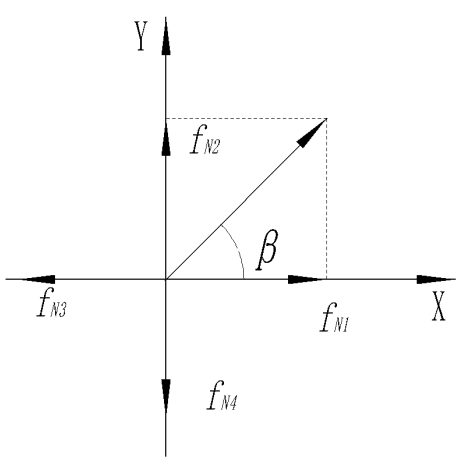

Figure 3. Horizontal component of four control lines

The horizontal component $f_{n}$ obtained by geometric calculation is:

$$
f_{\mathrm{N}}=f_{1} \cos \left(\arcsin \left(\frac{s}{l_{\mathrm{ab}}-\frac{R t \pi \omega}{180^{\circ}}}\right)\right)
$$

\section{Force Feedback Balance Model}

The wire rope generates a horizontal component force $\mathrm{fN}$ to the hanging plate in the horizontal direction, and the horizontal component forces of the four wire ropes are evenly distributed at $90^{\circ}$, and a plane rectangular coordinate system is established with the center point as the center, as shown in Fig. 3 below. When $f_{\mathrm{N} 1}=f_{\mathrm{N} 2}=f_{\mathrm{N} 3}=f_{\mathrm{N} 4}$, the resultant force is zero, and at this time, it is in an equilibrium state, and the wire control rope is in a tension state. When two horizontal components are increased, the lifting disk moves along the direction of resultant force of two changing components, and the moving direction Angle $\beta$ can be expressed as:

$$
\beta=\arctan \frac{\Delta f_{N_{2}}}{\Delta f_{N_{1}}}
$$

Where $\Delta f_{N 2}$ and $\Delta f_{N 2}$ represent the increase in the horizontal component force, respectively. When there are $\mathrm{n}$ line control ropes evenly distributed on the hanging plate, the center point of the force is taken as the center, and the direction of the $f_{N i}$ is the positive direction of the $\mathrm{X}$ axis, as shown in Fig. 4.The $\mathrm{N}$ horizontal component forces divide the plane into $\mathrm{n}$ angles, each angle is $\theta=2 \pi / \mathrm{n}$ ( $\mathrm{n}$ is even number), In the $\mathrm{N}$ horizontal component force diagrams, the two adjacent component forces are selected for analysis, and the serial numbers $\mathrm{n}$ of the two consecutive component forces are respectively $a$ and $b$. The Angle adjustment equation of line control rope can be obtained, as shown below:

(1) $\Delta f_{\mathrm{Na}}=\Delta f_{\mathrm{Nb}}, \beta=\left(\mathrm{a}-\frac{3}{2}\right) \frac{2 \pi}{n} \quad(\mathrm{n}>\mathrm{a}>\mathrm{b}>1$, a and $\mathrm{b}$ are two adjacent numbers). 
(2)

$$
\begin{aligned}
& \Delta f_{\mathrm{Na}}>\Delta f_{\mathrm{Nb}}, \quad \beta=\frac{2 \pi}{n}(\mathrm{a}-1)-\arcsin \left(\sin \left(\pi-\frac{2 \pi}{\mathrm{n}}\right) \Delta f_{N_{b}}\left(\sqrt{\Delta f_{N_{\mathrm{a}}}{ }^{2}+\Delta f_{N_{b}}{ }^{2}-2 \Delta f_{N_{\mathrm{a}}} \Delta f_{N_{b}} \cos \left(\pi-\frac{2 \pi}{n}\right.}\right)^{-1}\right) \\
& \left.\Delta f_{\mathrm{Na}}<\Delta f_{\mathrm{Nb}}, \quad \beta=(\mathrm{a}-2) \frac{2 \pi}{n}+\arcsin \left(\sin \left(\pi-\frac{2 \pi}{n}\right) \Delta f_{N_{a}}\left(\sqrt{\Delta f_{N_{\mathrm{a}}}{ }^{2}+\Delta f_{N_{b}}{ }^{2}-2 \Delta f_{N_{\mathrm{a}}} \Delta f_{N_{b}} \cos \left(\pi-\frac{2 \pi}{n}\right.}\right)\right)^{-1}\right)
\end{aligned}
$$

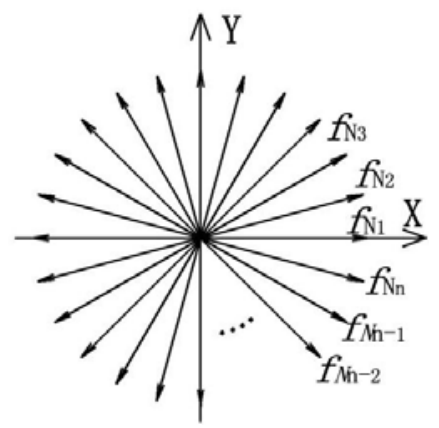

Figure 4. Horizontal component diagram of $\mathrm{N}$ line control rope

\section{An Analysis of the Leveling Motion of the Hanging Plate by Wire Control}

\section{Description of the Hanging Plate Posture}

From the perspective of robotics, the four-wire rope control system is equivalent to a four-cable-driven under-constrained parallel robot. The posture of the hanging plate is jointly determined by the length of the wire rope and the static equilibrium condition of the sling under the action of gravity. Based on the linear control system, the spatial position of the hanging plate center is calculated by geometric method, and the inverse kinematics model and the line length adjustment method of the line control rope are established to level the hanging plate with weight bucket.

The tilt Angle of the space of the sling is measured by an electronic level, and the tilt Angle and tilt azimuth are measured by using the pendulum principle, combined with sensor calculation and microcomputer measurement technology. As shown in Fig. 5, the $\mathrm{P}_{1}$ plane represents the horizontal hanging plate, the $\mathrm{P}_{2}$ plane represents the inclined hanging plate, and the radius of the disc is $\mathrm{r}, \mathrm{m}$ and $\mathrm{n}$ are the inclined azimuth angle and the inclined angle respectively. The inclined angle takes the positive direction of the $\mathrm{Y}$ axis as the starting reference direction and the clockwise direction as the positive direction. The space vector of $\mathrm{OQ}_{1}$ in the oxyz coordinate represents $\mathrm{PoQ}_{1}=[\mathrm{rs} \alpha,-\mathrm{rc} \alpha, 0]^{\mathrm{T}}$, and the space vector of $\mathrm{OQ}_{2}$ in the oxyz coordinate system represents $\mathrm{P}_{\mathrm{OQ}}=[\mathrm{rc} \beta \mathrm{s} \alpha,-\mathrm{rc} \alpha \mathrm{c} \beta,-\mathrm{rs} \beta]^{\mathrm{T}}$, where $\mathrm{s}()$ denotes sinusoidal operation and $\mathrm{c}()$ denotes cosine operation.

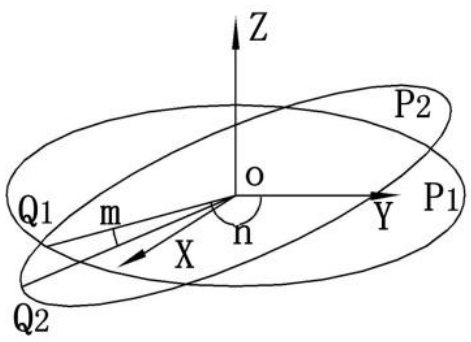

Figure 5. Inclination Angle and inclination Angle of the hanging tray

\section{Inverse Kinematics Model}

The Fig. 6 shows the schematic diagram of inverse kinematics analysis of the hanging tray. In the figure, $\mathrm{m}_{1} \sim \mathrm{m}_{4}$ represents the connection point between the wire rope and the hanging plate, and $\mathrm{n}_{1} \sim \mathrm{n}_{4}$ represents the fixed suspension point on the beam frame. The fixed reference coordinate system $\mathrm{O}_{\mathrm{xyz}}$ is defined at the starting center of the circular $\mathrm{n}_{1} \sim \mathrm{n}_{4}$ and the relative coordinate system $\mathrm{P}_{\text {noa }}$ is established on the hanging plate at the fixed point $\mathrm{P}$. Therefore, the center position of the hanging plate can be represented as $\mathrm{X}=[\mathrm{x}, \mathrm{y}, \mathrm{z}]^{\mathrm{T}}$, which is the coordinate of fixed point $\mathrm{P}$ in the fixed coordinate system. The attitude of the hanging plate can be expressed as $\boldsymbol{\theta}=\left[\varphi_{\mathrm{n}}, \varphi_{\mathrm{o}}, \varphi_{\mathrm{a}}\right]^{\mathrm{T}}$, where $\varphi_{\mathrm{n}}$ is 
yaw angle,$\varphi_{\mathrm{o}}$ is pitch angle and $\varphi_{\mathrm{a}}$ is rolling angle, they are the three rotation angles of the hanging plate with the relative coordinate system $P_{\text {noa. }}$

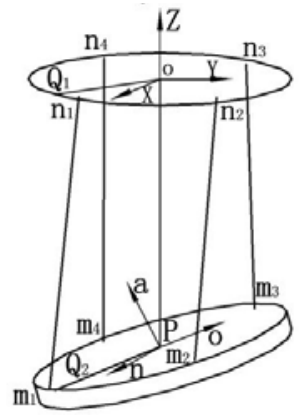

Figure 6. Schematic diagram of inverse kinematics analysis of the hanging tray

In the spatial coordinate representation, the transformation matrix along the $\mathrm{n}, \mathrm{o}$, a axis are: $\operatorname{Rot}\left(\mathrm{n}, \varphi_{\mathrm{n}}\right)=\left[\begin{array}{ccc}1 & 0 & 0 \\ 0 & c \varphi_{\mathrm{n}} & -s \varphi_{\mathrm{n}} \\ 0 & s \varphi_{\mathrm{n}} & c \varphi_{\mathrm{n}}\end{array}\right], \operatorname{Rot}\left(\mathrm{o}, \varphi_{o}\right)=\left[\begin{array}{ccc}c \varphi_{\mathrm{o}} & 0 & s \varphi_{\mathrm{o}} \\ 0 & 1 & 0 \\ -s \varphi_{\mathrm{o}} & 0 & c \varphi_{\mathrm{o}}\end{array}\right], \operatorname{Rot}\left(\mathrm{a}, \varphi_{a}\right)=\left[\begin{array}{ccc}c \varphi_{a} & -s \varphi_{\mathrm{a}} & 0 \\ s \varphi_{\mathrm{a}} & c \varphi_{\mathrm{a}} & 0 \\ 0 & 0 & 1\end{array}\right]$. Change matrix is $T(\theta)=\operatorname{Rot}\left(\mathrm{a}, \varphi_{a}\right) \operatorname{Rot}\left(\mathrm{o}, \varphi_{o}\right) \operatorname{Rot}\left(\mathrm{n}, \varphi_{\mathrm{n}}\right)=\left[\begin{array}{cccc}\mathrm{n}_{x} & o_{x} & a_{x} & o \\ n_{y} & o_{y} & a_{y} & o \\ n_{z} & o_{z} & a_{z} & 0 \\ 0 & 0 & 0 & 1\end{array}\right]$, Obtained: $\left[\begin{array}{c}r c \beta s \alpha \\ -r c \alpha c \beta \\ -r \sin \beta \\ 1\end{array}\right]=\left[\begin{array}{cccc}\mathrm{n}_{x} & o_{x} & a_{x} & o \\ n_{y} & o_{y} & a_{y} & o \\ n_{z} & o_{z} & a_{z} & 0 \\ 0 & 0 & 0 & 1\end{array}\right]\left[\begin{array}{c}r s \alpha \\ -r c \alpha \\ 0 \\ 1\end{array}\right]$

The equation obtained above is the suspension tray attitude conversion matrix, and the deflection Angle of $\varphi_{\mathrm{n}}, \varphi_{\mathrm{o}}$ and $\varphi_{\mathrm{a}}$ can be solved by calculating this equation.

When the hanging plate just lifts the weight bucket, the wire control rope is in a relaxed state, and for the hanging plate position $(\boldsymbol{x}, \boldsymbol{\theta})$, the coordinates of the point $\mathrm{m}_{\mathrm{i}}(\mathrm{i}=1,2,3,4)$ under the fixed coordinate system $\mathrm{O}_{\mathrm{xyz}}$ are $\mathrm{Xmi}=[\mathrm{Xmi}, \mathrm{Ymi}, \mathrm{Zmi}]^{\mathrm{T}}$.

We can obtained $\mathrm{P}_{\mathrm{mi}}=T(\theta) \mathrm{Xmi}_{\mathrm{mi}} \mathrm{X}_{\mathrm{mi}}=\mathrm{X}+\mathrm{P}_{\mathrm{mi}}$. Where $\mathrm{X}$ is the coordinates of the pendant in the $\mathrm{O}_{\mathrm{xyz}}$ fixed coordinate system, $\mathrm{X}_{\mathrm{mi}}$ is the position in the relative coordinate system $\mathrm{P}_{\text {noa }}$ before the pendant rotation, $\mathrm{P}_{\mathrm{mi}}$ is the coordinates of the pendant relative to the relative coordinate system $\mathrm{P}_{\text {noa }}$ before the tilt, and $\mathrm{T}(\boldsymbol{\theta})$ represents the rotational transformation matrix of the relative coordinate system $\mathrm{P}_{\text {noa }}$ relative to the fixed coordinate system $\mathrm{O}_{\mathrm{xyz}}$. Therefore, the distance between point $\mathrm{M}_{\mathrm{i}}$ and $\mathrm{N}_{\mathrm{i}}$ can be expressed as:

$$
l_{\text {mini }}=\mid n_{i} \text {-X-Pmi } \mid
$$

Where $\mathrm{N}_{\mathrm{i}}$ is the coordinate in $\mathrm{O}_{\mathrm{xyz}}$ in the fixed coordinate system. The formula is the relationship between the attitude position angle of the hanging plate and the length of the line control rope. Through this formula, The length difference between the line control rope and the line control rope at the leveling of the hanging plate can be calculated as $\Delta l_{i}$, where $i=1,2,3,4$. And the average of the four length differences is calculated as $\overline{\Delta l}$. If $\Delta l_{\mathrm{i}}>0$, indicating that the line control rope is in a relatively loose state, and the line control rope is decentralized $|l|=\left|\Delta l_{\mathrm{i}}-\overline{\Delta l}\right|$ unit length, at this time $l>0$;. If $\Delta l_{\mathrm{i}}<0$, indicating that the line control rope is in a relatively tight state, the line control rope is pulled up $|l|=\left|\Delta l_{\mathrm{i}}-\overline{\Delta l}\right|$ unit length, at this time $l<0$.

In summary, the reverse kinematics model of the cable control rope leveling can be expressed as:

$$
\begin{cases}l>0 & \left(\Delta l_{\mathrm{i}}>0\right) \\ l<0 & \left(\Delta l_{\mathrm{i}}<0\right)\end{cases}
$$

\section{Simulation and Experimental Analysis}

In order to test the capability of lifting plate force feedback balance and lifting plate attitude leveling of wire-controlled balancing mechanism, according to the inverse kinematics model and force feedback leveling model of lifting plate balancing mechanism established in this paper, the anti-sway and attitude leveling methods above were verified by establishing the cable model of 
Adams virtual simulation software cable module. The simulation model is shown below Fig. 7 .

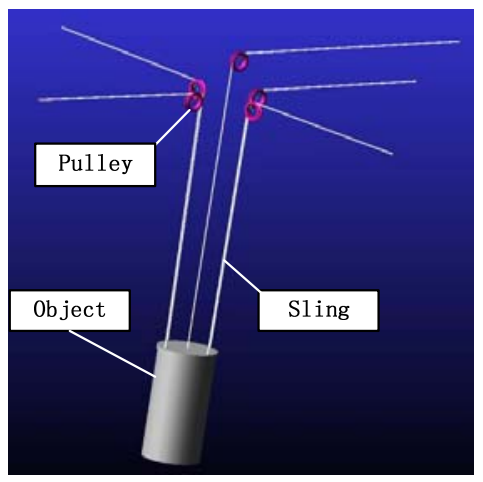

Figure 7. Diagram of Adams sling model

\section{Line Control Leveling Test}

The relevant parameters of the wire control and leveling lifting mechanism: the diameter of the main rope is $9 \mathrm{~mm}$, the diameter of the wire rope is $5 \mathrm{~mm}$, the lifting depth is set to be $3 \mathrm{~m}$, the mass of the weight bucket and tray is $500 \mathrm{~kg}$, and the cable material is steel which is installed in the cable module. The pulley diameter is set to be $200 \mathrm{~mm}$, The diameter of the rope is set to be $5 \mathrm{~mm}$ and $9 \mathrm{~mm}$ respectively. In establishing the pulley and the rack position using the location/orientation of LOC_RELATIVE_TO function, set its position as the $(0,0,0)$.

The simulation time is set to be $40 \mathrm{~s}$, the step length is set to be 100 , and the lifting speed of five wire ropes is set to be $50 \mathrm{~mm} / \mathrm{s}$. When the experiment is set to lift $5 \mathrm{~s}$, give the hanging plate a horizontal force with a bias angle of 30 and a size of $40 \mathrm{~N}$. Reacted at the line control rope of 5.6s, and the force simulation function was set to STEP(time, 5.5, 0, 5.6, 40) +(time, 6, 0, 6.5, -40).

In the process of lifting, when the feedback force is controlled wirelessly, the relation between the lifting disk offset and time is shown in Fig. 8.

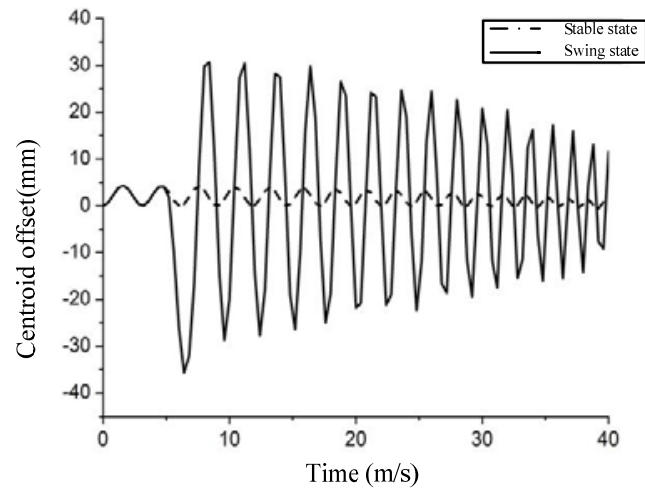

Figure 8. Center of mass displacement of hanging plate (no feedback force)

After adding the feedback force of wire control, the relation between the sling offset and time is shown in Fig.9.

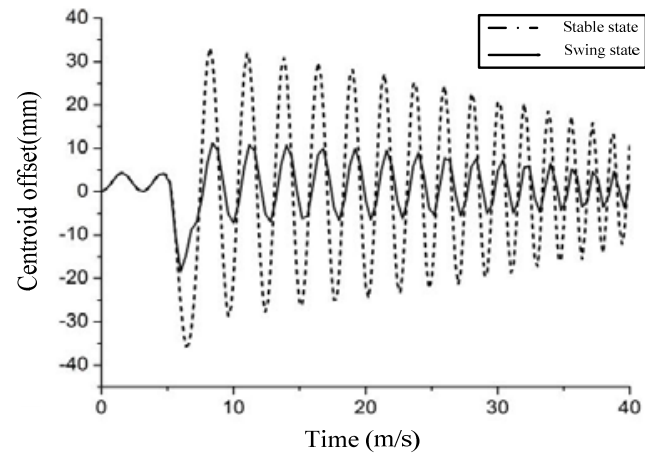

Figure 9. Center of mass deviation (add feedback force) 
As can be seen from Fig. 8 and 9, when there is no feedback force in Fig.10, the hanging plate reaches a maximum offset of $36.4 \mathrm{~mm}$ at $6.4 \mathrm{~s}$, and the self-balancing takes a long time. After adding the feedback force in Fig.13, the maximum offset of the tray is $17.5 \mathrm{~mm}$, and the $11 \mathrm{~s}$ offsetis reduced to a safe offset interval below $12 \mathrm{~mm}$, and the linear balance time is $5.4 \mathrm{~s}$.

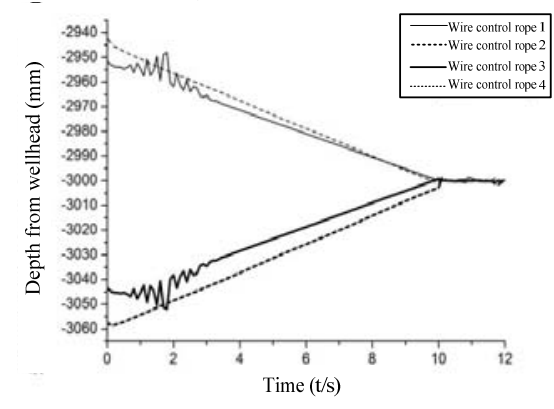

Figure 10. Relative depth of four wire rope connection points on the hoist

\section{Hanging Plate Attitude Leveling Test}

The steps of the hanging plate leveling experiment are as follows: First, the lower part of the hanging plate is 3 meters away from the wellhead; Changing the attitude of the suspension, the tilt azimuth and tilt angle of the suspension were measured at $18.14^{\circ}$ and $74.16^{\circ}$, respectively. According to the reverse kinematics model, it can be obtained that the deviation angle $\varphi_{\mathrm{n}}$ is 17.3, the pitch angle is $\varphi_{o}$ is $14^{\circ}$, the rolling angle $\varphi_{\mathrm{a}}$ is -14.5 , and the change quantities of the line control rope are $56.11 \mathrm{~mm}, 47.36 \mathrm{~mm},-44.39 \mathrm{~mm}$, and $-59.14 \mathrm{~mm}$, respectively. The simulation time was $12 \mathrm{~s}$, and the step length was 100 , which describes the relationship between the length change and time of the rope segment during the attitude leveling process of the four wire control ropes.

As can be seen from Fig. 10, during the leveling process, the line control rope made two actions: lifting and lowering, respectively, and at 10s, the four starting points approached the depth of the wellhead by nearly $3000 \mathrm{~mm}$. At this time, the plane of the hanging plate is parallel to the wellhead plane, and the posture of the hanging plate completes leveling. The relative deviation of four points tends to $\pm 1 \mathrm{~mm}$.

\section{Conclusion}

The force feedback leveling model of the hanging plate is established. When the swaying direction is measured, the binding force opposite to the swaying direction can be obtained by changing the tension force of the wire control rope with the force feedback leveling model, and the swaying direction can be effectively reduced.

The inverted motion model is established. When the slant Angle and the slant direction Angle of the sling are known, the displacement of four strings can be obtained through the analysis of the reverse kinematics model, and the displacement of the strings can be changed to achieve the attitude leveling of the sling. The simulation software Adams is used to verify the correctness of the model and its good attitude leveling ability.

\section{Acknowledgement}

Hunan Provincial Natural Science Foundation Funded Project (2015JJ5023)

Hengyang City Industry-University Research Project (2017KJ143)

\section{References}

[1] Zhu Bao, Yu Yongsheng, Kang Ningmin, Mu Zhongbo, The Researsh of Arithmetic of Level Angle Decomposition When Launcher Turned Around, J.Journal of Astronautic Metrology and Measurement.2011,31(02):5-7. 
[2] Ni Jiangsheng, Zhai Yujian.The Self-Leveling Method for Six Supports Hydraulic Platform on Stationary Base,J. Journal of Southeast University.1996,(02):74-80.

[3] Zhang Fang. Study on Leveling Control System of High Precision Platform, D.North University of China.2008.

[4] Yu Y, Yi J, Li C, et al. Fuzzy logic based adjustment control of a cable-driven auto-leveling parallel robot, C.International Conference on Intelligent Robots and Systems. IEEE, 2009: 2102-2107.

[5] ShaoXingguo, Zhu Zhencai, Cao Guohua, Li Yilei. Auto-leveling control for sinking winch mechanism and experimental validation, J.journal of china coal society, 2012, 37(03):528-532

[6] Zhang Fuming. The design of five point synchronous lifting system based on PLC,D. Hefei University of Technology.2017.

[7] Du Wenzheng, Tong Guolin, Qiang Baomin, Wang Weihui, Xie Zheng.Research of the position and anti-swing control for bridge crane based on fuzzy adaptive PID controller, J.Manufactuing Automation. 2012, 34(22):8-11.

[8] Wang Xiaojun, Shao Huihe. Fuzzy Logic-based Anti-Swing and Position Control for Bridge Cranes, J.Journal of system simulation. 2005(04):936-939

[9] Wang Keqi.Study of Anti-Swing and Position Control for Bridge Cranes, J.Journal of System Simulation .2007(08):1799-1802. 